\title{
System for 3D Localization and Synchronization of Embedded Wireless Sensor Nodes Based on AMR Sensors in Industrial Environments
}

Carrella, Stefano, Iswandy, Kuncup, and König, Andreas

Institute of Integrated Sensor Systems, TU Kaiserslautern

Erwin-Schrödinger-Straße Geb. 12, 67663 Kaiserslautern

\section{Introduction}

In the last decade, wireless sensor networks (WSN) have gathered a lot of attention as important research and application domain. In many applications, e.g., ambient intelligence (Aml), medical, military, and monitoring of industrial processes, wireless sensor nodes are used for measuring and transmitting information. For many of those applications, knowledge of the sensor node position is mandatory. For the localization of sensor nodes, technologies based on radio are widely used. However, there are some constraints imposed by the environment, which hinder a reliable localization via radio, e.g., steel and liquids. In particular for those environments, e.g., in the food or chemical industry, where liquid filled containers are used, a localization concept based on magnetic quasi DC fields has been developed and evaluated by using wired sensor nodes. Precise clocks are mandatory to deploy aforementioned localization concept to wireless sensor nodes. While choosing the time reference, the trade-off between accuracy and power consumption must be taken into account. In particular for wireless sensor nodes with a restricted amount of energy, quartz crystals with tolerances down to 10ppm or RC oscillators with even higher tolerances are widely used. More accurate time references would exceed the maximum tolerable power consumption for many applications. Thus, a need of synchronization rises out of the tolerances of used time references. The constraints, put by industrial environments, not only have an impact on the localization, but also on the synchronization. Therefore, most of the existing synchronization methods are not suitable and a new concept of synchronization, based on the magnetic quasi DC fields, has been developed and is proposed in this paper.

This paper is organized as follows. The related work is presented in section 2, where the localization concept based on magnetic quasi DC fields is described briefly. Then, existing synchronization methods are discussed. In section 3 , the proposed synchronization concept is elaborated. The implementation and the evaluation of the concept are given in section 4. Section 5 summarizes the paper and gives an overview of future work.

\section{Related Work}

\subsection{Localization concept}

In the last decade, concepts and technologies for the localization of wireless sensor nodes has been developed. However, there are some environments, e.g., some industrial environments, which hinder a reliable localization via radio due to attenuation of radio frequency and thus, hinder the applicability of most already existing technologies, e.g., such based on ultrasonic or light, due to attenuation, reflection, and opaqueness. To overcome many constraints imposed by industrial environments on the localization, a concept based of magnetic fields has been developed.

The magnetic fields, on which our proposed localization concept is based, are emitted by core-less coils, which are encompassing the measurement volume, i.e., the possible sensor location. The coils are sequentially switched and the magnetic field strength emitted by the coils is measured by a tri-axial AMR sensor, which is integrated in the sensor nodes. Additionally, each coil has three switching statuses (fig. 2 top). First, a certain magnetic field strength is emitted in one direction, than, the same field strength is emitted in the opposite direction, and finally, no field is emitted. Between each switching, there is a certain delay that enables the establishment of DC plateaus of magnetic field strength. The measurements of the 
field strength take place for the first and for all second plateaus, respectively. The values are subtracted from each other for each coil, to remove offsets, in particular the earth magnetic field offset. The differences are used to compute the distances from the sensor node to each coil. These distances are used by a range-based algorithm, e.g., triangulation or Sammon's mapping, to compute the sensor node position. A proof of principle is given in [1]. In [2], the applicability of our approach to stainless steel containers filled by water is shown. The range of localization has been scaled to a scale required for the localization in medium size industrial containers [3]. For the applicability of this localization concept to wireless sensor nodes with restricted size and power and a need of high resolution, AMR sensor elements are used. An overview of magnetic field sensors, including the achievable resolution, is given in $[4,5]$. Additionally, to ensure low power consumption, duty-cycling of sensors should be supported by, e.g., a current source that is included in an instrumentation amplifier [1].

Due to missing capabilities to emit and measure the field synchronously, cable-connected sensor nodes have been applied in the proof of principle demonstrator. To extend the localization concept to wireless sensor nodes, synchronization capabilities are required. The synchronization must work in all environments, the localization concept is used for. The error in time must be smaller than half a DC plateau length. Otherwise, the magnetic field strength of the DC plateaus are not measured and the localization fails. Currently the coils are switched every $62.5 \mathrm{~ms}$. Future implementations might have only $5 \mathrm{~ms}$ DC plateau duration and require a clock deviation smaller than $2.5 \mathrm{~ms}$. Additionally, for the date of measurements of other values, e.g., temperature, and for wake up times, precise clocks are advantageous, as well.

\subsection{Synchronization}

Due to non-identical initialization of clocks and due to the limitations of accuracy of time references, two clocks differ from each other. The non-identical initialization might be caused by, e.g., a different amount of time to get the clocks stable or by different delays for the propagation of the signal, used for the initialization. While running, there is a drift between the clocks due to non-identical frequencies of time references. For instance, the frequency of quartz crystals hardly has less than $\pm 10 \mathrm{ppm}$ deviation due to production tolerances. In addition, changing temperature and aging increase the tolerances of those time references. Even though, there are high accurate time references, due to restrictions in terms of power consumption, size, and cost, in wireless sensor nodes, $32.768 \mathrm{kHz}$ quartz crystals or RC oscillators are widely used as time references for the real time counter. While quartz crystals have a power consumption of a few $\mu \mathrm{W}$, more accurate temperature compensated oscillators already require several $\mathrm{mW}$. Usually, the more accurate a clock is, the more power it consumes. In [6] an overview of different time references and their power consumptions are given.

The synchronization of wireless sensor nodes, already has been discussed in recent years. In, e.g., [7], the synchronization in wireless networks is discussed in general. While most synchronization methods for wireless networks are based on radio, e.g., [8], there are some based on other media, e.g. acoustic signals [9]. However, for the presented localization concept, synchronization via radio or acoustic signals is not suitable because of the environment, the localization concept has been designed for. Pragmatically, the already expended resources and efforts for the localization can be exploited for the synchronization and would result either in both a reliable localization and synchronization or none of both. A single particular synchronization method based on magnetic fields can be found in [10]. The synchronization concept presented in [10] uses the magnetic fields, which are radiated from the AC power lines. Due to the size of the container and due to the magnetic fields emitted by the coils used for the localization and by industrial equipment, this approach does not appear suitable for our application scenario. In [10] the synchronization is limited to stationary devices, which hinders the applicability in moving wireless sensor nodes. Therefore, an alternative way of synchronization based on magnetic fields is needed and the localization concept has been extended by a synchronization concept that reuses the existing hardware resources of the localization, i.e., the sequentially and ternary switched magnetic fields and the magnetic sensors.

\section{Synchronization Concept}

To reduce the time difference between the clock giving the time reference for the switching of the field generating coils, and the clock giving the time reference for the measurements of the magnetic field strength, two approaches are used. First, the frequencies of the clocks are calibrated by adjusting the top value of the real time counter. In consequence, the drift is reduced. Widely used $32.768 \mathrm{kHz}$ quartz 
crystals may have frequency tolerances down to $10 \mathrm{ppm}$. By adding a calibration of the real time counter, the tolerance can easily be reduced to $2 \mathrm{ppm}$ for environments with small changes of temperature. In figure 1, the drift between two clocks is illustrated, where the black curve shows the deviation for two clocks of which one was calibrated to the frequency of the other and the blue curve shows the drift that would exist without calibration. In sensor nodes with temperature sensors, the measured temperature can be used in addition, to compensate the effect of the temperature to the frequency of the quartz crystal. However, the calibration of the speed does not completely hinder the drift of clocks, due to, e.g., imprecise measurement of temperature or noise in the power line, and an additional mechanism is required to compensate this. This second mechanism is the actual synchronization.

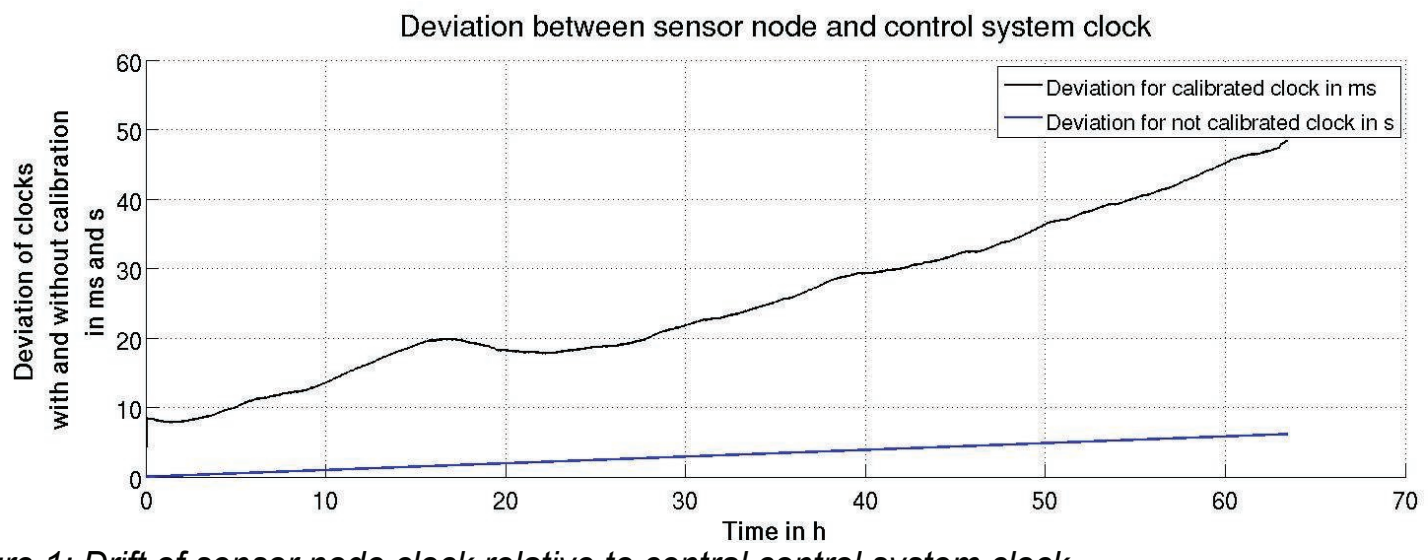

Figure 1: Drift of sensor node clock relative to central control system clock.

At the very beginning, the clocks are synchronized via state-of-the-art methods. For sensor nodes employed in industrial applications, this synchronization may be done via radio, even before the sensor nodes are deployed in the actual application environment. In the application environment, there are the magnetic fields that are generated for the localization. Between these quasi-DC plateaus, there are edges (fig. 2). These edges are controlled by the central control system and used by the sensor nodes as time stamps, to which the sensor nodes can synchronize to. Thus, there is a mono-directional synchronization that can be considered as master slave relation, where the time of the master (central control system) is used by the slaves (wireless sensor nodes). The magnetic field strengths measurable at DC plateau centers depend on the sensor node position. To achieve a reliable synchronization, only the coils that emit a significant field strength value at the sensor node's position can be employed for the synchronization. Therefore, the edge, each sensor node synchronize to, is chosen autonomously by each sensor node. That edge belongs to, e.g., the first coil of each localization cycle that emits an above threshold field strength value. For the selected coil, after the measurement of the field strength of the second DC plateau, the ADC keeps running and samples the edge from the second DC plateau to the resting plateau of the selected coil (fig. 2). The index of the sample corresponding to the beginning of the edge is detected by an algorithm. Based on this sample index $s_{i}$ (fig. 2) and the ADC sampling speed, the delay between the start of the ADC and the beginning of the edge is calculated. The actual delay is compared to the nominal value of delay, i.e., the expected edge position, and the time difference between the clocks is calculated. The time reference of the sensor node is corrected according to the calculated time difference.

Additionally, based on the assumption of a permanent difference in clock frequency, the frequency of the sensor node clock might be adjusted. This adjustment would be an enhancement of aforementioned calibration of clock speed by, e.g., adjusting the top value of the real time counter.

The proposed synchronization concept at the current state of the work still faces two limitations. First, due to the uniformity of the switching pattern, the sensor node cannot distinguish the single coils, except based on the time. Thus, if the deviation of time amounts to the duration of one coil cycle, the sensor node is not able to detect this deviation. Second, if the drift of time is bigger than half a DC plateau duration, faulty measurements for the localization are taken while there is no DC plateau and the synchronization might fail to be triggered. 


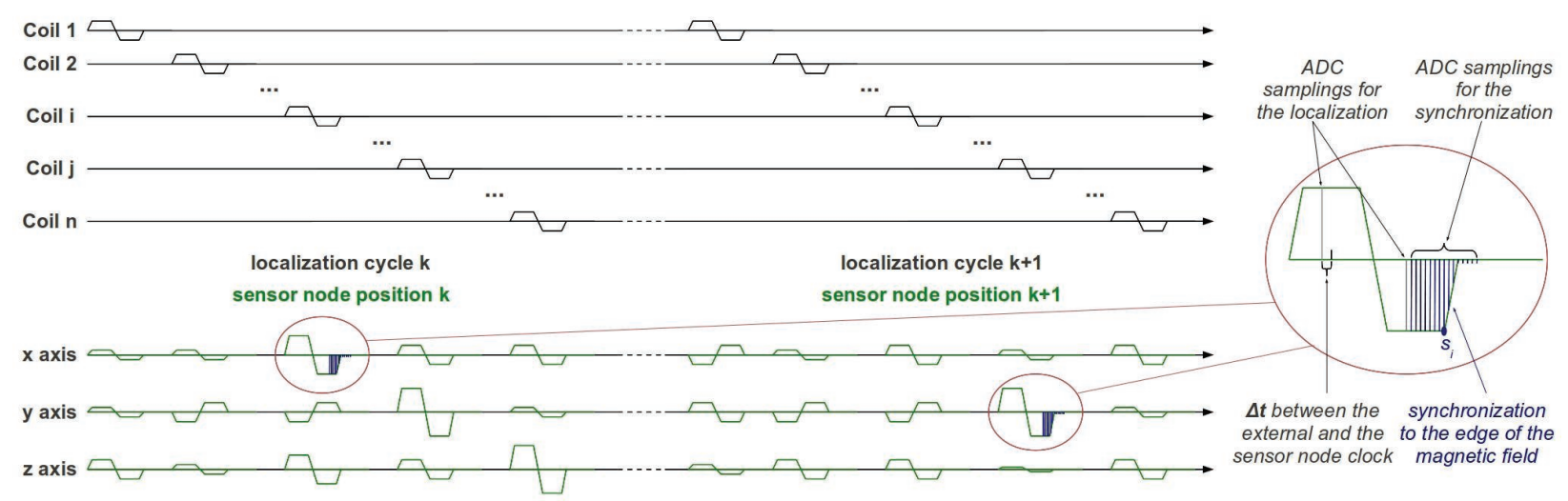

Figure 2: Coil switching pattern and use of the resulting emitted magnetic field strength in the sensor node location for the synchronization.

\section{Implementation and Results}

The set-up of the proof of principle demonstrator is shown in figure 3. The switching of the magnetic field is controlled by a MCU Xmega256A3, which uses $32.768 \mathrm{kHz}$ quartz crystal with a nominal deviation of $\pm 10 \mathrm{ppm}$ as time reference. A Gossen Konstanter $84 \mathrm{~T} 32 \mathrm{R} 40$ supplies $3 \mathrm{~A}$ through mechanical relays to the coils with a diameter of $25 \mathrm{~cm}, 230$ windings, a length of $5 \mathrm{~cm}$ and a wire gauge of $0.63 \mathrm{~mm}$. The sensor node consists of a tri-axial AMR sensor with three AFF755 AMR sensor elements and three AD8290 amplifiers, a MCU Xmega256A3, and a $32.768 \mathrm{kHz}$ quartz crystal with a nominal deviation of $\pm 10 \mathrm{ppm}$ as time reference. In addition, an output pin of each MCU is connected to an oscilloscope.

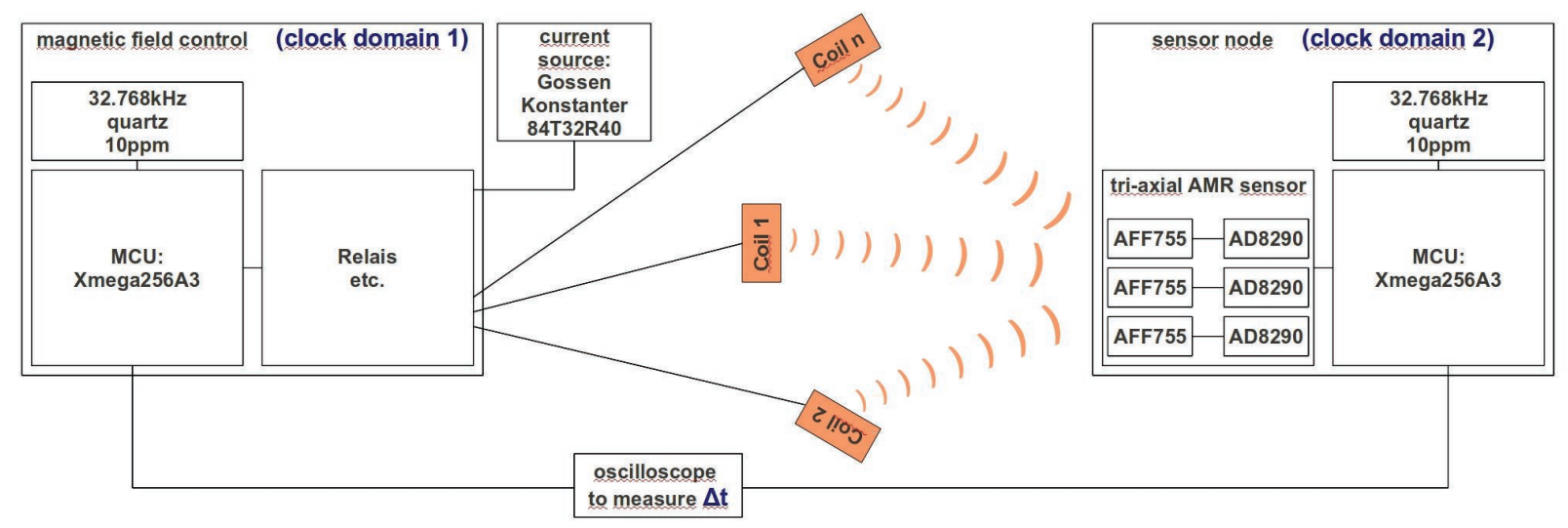

Figure 3: Set-up for the synchronization.

The output pins of both the MCUs are toggled twice per localization cycle, once before and once after synchronization. First, for a period of $20 \mathrm{~min}$, no field was emitted, to avoid synchronization and to measure the drift between both clocks. The sensor node clock was approximately $2.3 \mathrm{~ms}$ per minute faster than the one, used for the generation of the magnetic fields. Due to the need of a certain deviation of the clocks for testing the method, the aforementioned calibration of clocks by adjusting the maximum value of the real time counters was not applied. The calibration of clocks by adjusting the top value of the real time counter was tested previously. The result for a period of more than $60 \mathrm{~h}$ is shown in fig. 1. In addition to the uncalibrated clocks, a localization and synchronization period of $2 \mathrm{~min}$ was used, which results in approximately $4.6 \mathrm{~ms}$ deviation of time per cycle. During the localization cycles, the coils are switched every $62.5 \mathrm{~ms}$. The rise and fall times of the edges for the synchronization was approximately $7 \mathrm{~ms}$. For both the DC plateaus, eight samples were taken for the standard localization and the mean value computed. For the first coil that emits a field strength value above the threshold, the ADC keeps running. The channel with the highest change in field strength of the tri-axial AMR sensor at a sampling frequency of $15.625 \mathrm{kHz}(8 \mathrm{MHz} / 512)$, which corresponds to a period of $64 \mu \mathrm{s}$. 1025 samples were taken 
and for practical reasons, from those, the edge value that corresponds to $50 \%$ of the maximum field strength value was searched (fig. 4). Based on the index of this $50 \%$-sample, the difference between the clocks was calculated and the value of the real time counter of the sensor node was corrected.

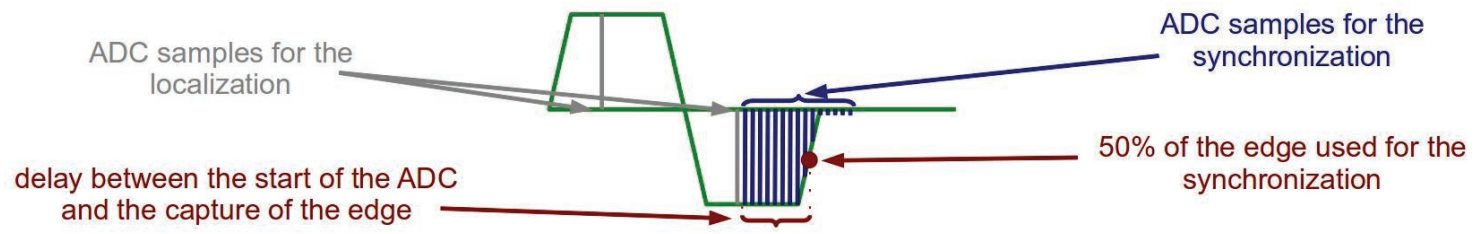

Figure 4: Capture of the edge deployed in the implementation.

The deviation of clocks was shown by an oscilloscope that received the signals of the output pins of both MCUs, which were toggled twice (fig. 5), once at the beginning of each period and a second time after synchronization. The second toggling was employed for the evaluation of the actual synchronization, while the first toggling of each period only served to verify that the clocks still have approximately $4.6 \mathrm{~ms}$ deviation per 2 minute cycle. For a moving sensor node, during 60min deployment time with 23 synchronizations, a mean synchronization error of $1.55 \mathrm{~ms}$ with $0.67 \mathrm{~ms}$ standard deviation could be determined. During the experiment, the sensor node was brought several times out of reach of the coils to prove the ability to synchronize even after previously failed synchronizations, where the maximum measured deviation before localization was $21.1 \mathrm{~ms}$ and still smaller than half a DC plateau length. The error that could be seen on the oscilloscope, arises partly from the delay between the capture of the edge and the output toggle that was between $0.8 \mathrm{~s}$ and $2 \mathrm{~s}$ (fig. 5). The delay between the capture of the edge and the output toggle depends on the coil selected for the synchronization. Additionally, the sampling frequency of the $A D C$ has a deviation up to $1 \%$. This sampling frequency has not been calibrated. $A$ measurement of the actual frequency has also not yet been done. In the future, knowledge of the actual sampling frequency can be considered by the algorithm and thus, the delay between the start of the ADC sampling and the edge can be calculated properly. However, by having only a small slot of time, where the ADC runs $(64 \mathrm{~ms})$, the effect was still in a tolerable range (maximum of $320 \mu \mathrm{s})$.
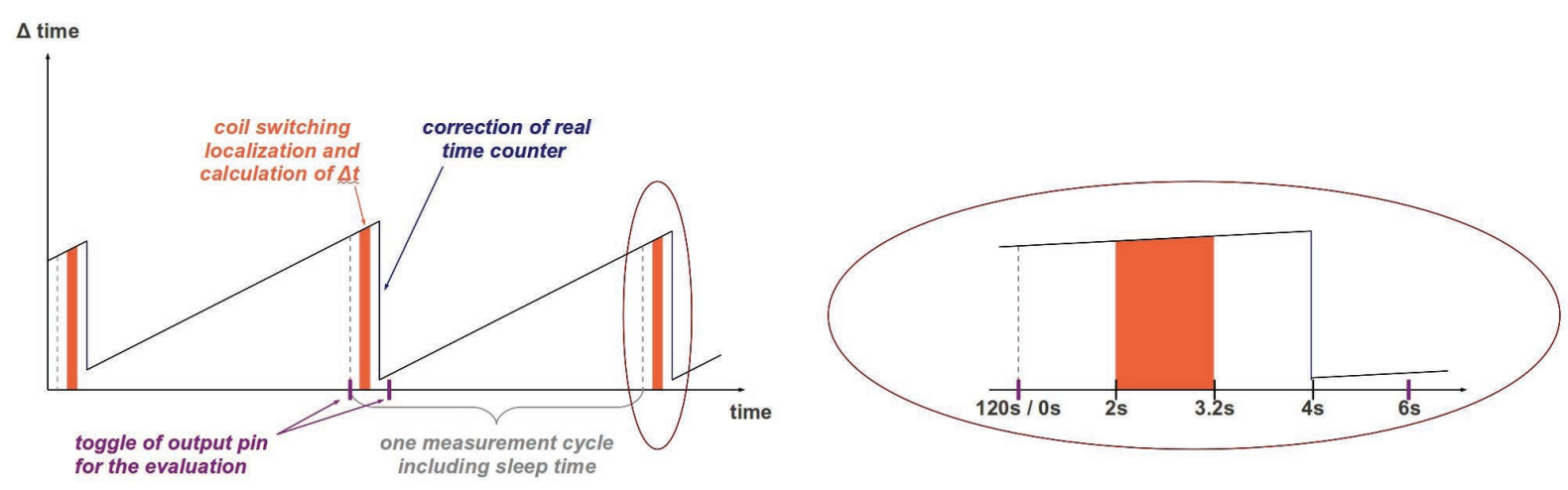

Figure 5: Timing diagram of the implementation.

\section{Conclusion and Future Work}

The localization method based on magnetic DC fields could successfully been extended by a synchronization method. The synchronization error $<2 \mathrm{~ms}$ is sufficient for a localization that uses DC plateau lengths of $62.5 \mathrm{~ms}$. A deviation of $2.3 \mathrm{~ms}$ per minute leads to the need of at least one synchronization in $10 \mathrm{~min}$.

By adding a calibration of the clock by, e.g., adjusting the top value of the real time counter, the drift in one measurement cycle can be reduced (fig. 1) and one synchronization in more than $1 \mathrm{~h}$ would be sufficient. By using the actual ADC sampling rate for calculating the delay, the correction can be more accurate. To enable a localization in industrial applications with 20 coils and within $300 \mathrm{~ms}$, a maximum 
plateau duration of $5 \mathrm{~ms}$ is aspired. Therefore shorter edges are required. A current source with faster regulation and faster switches based on modern power electronic devices might be used instead. For the steeper edges and shorter DC plateau lengths, the ADC sampling rate can be increased and a correction of time up to the resolution of the real time counter with the $32.768 \mathrm{kHz}$ quartz crystal, i.e., approximately $31 \mu \mathrm{s}$, might be reached, but still must be verified by future work.

Future work additionally will consider the possibility to modify the switching pattern of the field emitting coils to get a real time stamp and enabling the correction of deviations bigger than half a DC plateau length. The modification of switching pattern, might include the order of the coils, variations in the length of the DC plateaus, and different delays between different coils. Additional samples of the ADC might be required for detecting such non-regularities in the switching pattern. The synchronization concept proposed and evaluated can be combined with aforementioned modification of switching pattern. In cases the sensor node can detect meaningful field strengths, the ADC only keeps running after the trigger arising from the localization. In cases no meaningful field strength can be detected during the localization, the sensor nodes assume, they have a big deviation of time and keep the ADCs running for a long period of time to get the whole switching pattern and do a real synchronization. Robust, fault tolerant algorithms for proposed synchronization concept are matter of research as well. In particular, peaks due to noise or other magnetic fields should not effect the clock of sensor nodes by wrong synchronizations.

\section{Acknowledgement}

This work was supported by the Federal Ministry of Education and Research (BMBF) in the program mstAVS, PAC4PT consortium coordinated by microTEC Gesellschaft für Mikrotechnologie $\mathrm{mbH}$, project ROSIG grant no. 16 SV3604.

\section{References}

[1] Carrella, S., Iswandy, K., Lutz, K., König, A.: 3D-Localization of Low- Power Wireless Sensor Nodes Based on AMR Sensors in Industrial and Aml Applications. In: 15. ITG-/GMA-Fachtagung Sensoren und Messsysteme 2010, Nürnberg, pp. 522-529,May 2010.

[2] Iswandy, K., Carrella, S., König, A.: Intelligent Magnetic Sensing System for Low Power WSN Localization Immersed in Liquid-Filled Industrial Containers . In: Setchi, R. (Ed.) ; Jordanov, I. (Ed.) ; Howlett, R. J. (Ed.) ; Jain, L. C. (Ed.): Knowledge-Based and Intelligent Information and Engineering Systems Vol. 6277, pp. 361-370 ,Springer, 2010 (Lecture Notes in Computer Science).

[3] Iswandy, K., Carrella, S., König, A.: Localization System for Low Power Sensor Nodes Deployed in Liquid-Filled Industrial Containers Based on Magnetic Sensing. In: 24. Messtechnisches Symposium des Arbeitskreises der Hochschulleher für Messtechnik e.V., Hamburg, pp.108-121, Sept 2010.

[4] Lenz, J. E.: "A Review of Magnetic Sensors“. In: Proc. of the IEEE, vol. 78, no. 6, pp. 973-989, Jun. 1990.

[5] Lenz, J. E.; Edelstein, A. S.: "Magnetic Sensors and Their Applications". In: IEEE Sensors Journal, vol. 6, no. 3, pp.631-649, Jun. 2006.

[6] Sadler, B. M.: "Fundamentals of Energy-Constrained Sensor Network Systems". In IEEE A\&E Systems Magazine, vol. 20, no. 8, pp. 17-35, Aug. 2005.

[7] Simeone, O., Spagnoli, U., Bar-Ness, Y., Strogatz, S. H.: "Distributed Synchronization in Wireless Networks". In: IEEE Signal Processing Magazine, vol. 25, no. 5, pp. 81-97, Sept. 2008.

[9] Syed, A. A., Heidemann, J.: "Time Synchronization for High Latency Acoustic Networks". In $25^{\text {th }}$ IEEE International Conference on Computer Communications, pp. 1-12, Apr. 2006.

[8] Mock, M., Nett, E., Frings, R., Trikaliotis, S.: "Continuous Clock Synchronization in Wireless Real-Time Applications". In: 19 ${ }^{\text {th }}$ IEEE Symposium on Reliable Distributed Systems, pp. 125,132, Oct. 2000.

[10] Rowe, A., Gupta, V., Rajkumar, R.: "Low-power Clock Synchronization using Electromagnetic Energy Radiating from AC Power Lines". In: 7th ACM Conference on Embedded Networked Sensor Systems (SenSys), pp. 211-224, Nov. 2009. 